average screening rate in months prior to the use of Viz RECRUIT ICH Volume was 5.4 patients per month. After implementation, average screening rate increased to 7.6 patients per month. The average enrollment rate prior to the use of Viz ICH VOLUME was 0.29 patients per month, which increased to 0.82 after implementation. This equated to an increase in trial enrollment of $213 \%$.

Conclusions Artificial intelligence based programs such as Viz RECRUIT ICH Volume may help increase RCT screening and enrollment by identifying potential candidates automatically and alerting medical professionals quickly.

Disclosures B. Jankowitz: 2; C; stryker, medtronic. J. Davies: None. C. Schirmer: None. J. Day: None. O. Bibas: None. N. Levkovitz: None. E. Blanc: 5; C; Viz.ai. G. Pradilla: None.

\section{E-023 PRECLINICAL AND CLINICAL ROLE OF INTERLEUKIN-6 IN THE DEVELOPMENT OF DELAYED CEREBRAL VASOSPASM AND NEURONAL CELL DEATH AFTER SUBARACHNOID HEMORRHAGE: TOWARDS A POTENTIAL TARGET THERAPY}

${ }^{1} \mathrm{G}$ Agnoletto*, ${ }^{1} \mathrm{D}$ Croci, ${ }^{2} \mathrm{~S}$ Sivanrupan, ${ }^{2} \mathrm{~S}$ Wanderer, ${ }^{3} \mathrm{~A}$ Chiappini, ${ }^{2} \mathrm{~B}$ Grüter, ${ }^{2} \mathrm{~L}$ Andereggen, ${ }^{3} \mathrm{~L}$ Mariani, ${ }^{1} \mathrm{P}$ Taussky, ${ }^{4} \mathrm{~S}$ Marbacher. ${ }^{1}$ Neurosurgery, University of Utah, Salt Lake City, UT; ${ }^{2}$ BioMedical Research, University of Bern, Bern, Switzerland; ${ }^{3}$ Neurosurgery, University of Basel, Basel, Switzerland; " Neurosurgeyr and BioMedical Research, University of Bern, Bern, Switzerland

\subsection{6/neurintsurg-2021-SNIS.119}

Introduction Delayed cerebral vasospasm (DCVS), early brain injury (EBI), and delayed cerebral ischemia (DCI) are devastating complications after aneurysmal subarachnoid hemorrhage (SAH). Interleukin (IL)-6 seems to be a key interleukin in the inflammatory response after $\mathrm{SAH}$, and many studies describe a strong correlation between IL- 6 and worse outcome. The aim of this study was to systematically review preclinical and clinical studies that evaluated systemic and cerebral IL-6 levels after SAH and their relation to DCVS, neuronal cell death, and DCI.

Methods We conducted two systematic literature searches using PubMed to identify preclinical and clinical studies evaluating the role of IL-6 after SAH. Suitable articles were selected based on predefined eligibility criteria following the Preferred Reporting Items for Systematic Reviews and Meta-Analyses guidelines.

Results A total of 61 and 30 preclinical and clinical articles, respectively, were included in the systematic reviews. Of the preclinical studies in which IL-6 was measured in cerebrospinal fluid (CSF), parenchyma, and systemically, 100\%, 94.4\%, and $81.3 \%$, respectively, showed increased expression of IL-6 after SAH. Preclinical results were mirrored by clinical findings in which elevated levels of IL-6 in CSF and plasma were found after SAH, correlating with DCVS, DCI, and worse outcome. Only two preclinical studies analyzed the direct inhibition of IL-6, which resulted in reduced DCVS and neuronal cell death.

Conclusion IL-6 plays a fundamental role in the onset of DCVS and DCI after SAH in preclinical animal models and clinical studies. Its inhibition might have therapeutic potential to improve the outcome of SAH patients.

Disclosures G. Agnoletto: None. D. Croci: None. S. Sivanrupan: None. S. Wanderer: None. A. Chiappini: None. B. Grüter: None. L. Andereggen: None. L. Mariani: None. P. Taussky: None. S. Marbacher: None.

\section{E-024 ASPIRATION THROMBECTOMY ON ACUTE ISCHEMIC STROKE PATIENTS WITH A LOW ALBERTA STROKE PROGRAM EARLY COMPUTERIZED TOMOGRAPHY SCORE}

1J Fifi* ${ }^{2,3} \mathrm{~A}$ Hassan, ${ }^{4} \mathrm{O}$ Zaidat. ${ }^{1} / \mathrm{c}$ ahn School of Medicine at Mount Sinai, New York, NY; ${ }^{2}$ University of Texas Rio Grande Valley, Harlingen, TX; ${ }^{3}$ Valley Baptist Medical Center, Harlingen, $\mathrm{TX}_{i}{ }^{4}$ Mercy Health St. Vincent Medical Center, Toledo, $\mathrm{OH}$

\subsection{6/neurintsurg-2021-SNIS.120}

Introduction/Purpose For patients who receive endovascular therapy for acute ischemic stroke (AIS), a lower Alberta Stroke Program Early CT Score (ASPECTS) correlates with worse outcomes. However, AIS patients with a low ASPECTS may still have a better outcome after endovascular therapy than after best medical therapy. The purpose of this study was to

Abstract E-023 Table 1 Animal and human studies measuring interleukin-6 in cerebrospinal fluid, plasma/serum, parenchyma, and the cerebral vasculature

\begin{tabular}{|l|l|l|l|l|}
\hline & \multicolumn{2}{|l|}{ Animal (n=61) } & \multicolumn{2}{l|}{ Human (n=30) } \\
\cline { 2 - 6 } & $\begin{array}{l}\text { Number } \\
\text { (\%) of }\end{array}$ & Number (\%) of & Number & Number (\%) of \\
IL-6 measurement & studies & increase in IL-6 & studies & increase in IL-6 \\
\hline Cerebrospinal fluid (\%) & $14(22.9)$ & $14(100)$ & $17(56.7)$ & $17(100)$ \\
\hline Plasma/serum (\%) & $16(26.2)$ & $13(81.3)$ & $23(76.7)$ & $23(100)$ \\
\hline Parenchyma (\%) & $36(59)$ & $34(94.4)$ & - & - \\
\hline Cerebral vasculature (\%) & $7(11.4)$ & $7(100)$ & - & - \\
\hline
\end{tabular}

